\title{
Os Desafios da Insuficiência Cardíaca Ontem, Hoje e Amanhã, e os 20 Anos do DEIC
}

\author{
The Challenges of Heart Failure Yesterday, Today and Tomorrow and the 20 Years of DEIC
}

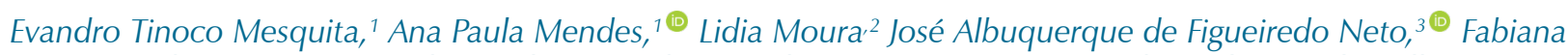
G. Marcondes-Braga, ${ }^{4}$ Fernando Bacal, ${ }^{4}$ Maria da Consolação Vieira Moreira, ${ }^{5}$ Nadine Oliveira Clausell ${ }^{6}$

Universidade Federal Fluminense Hospital Universitário Antônio Pedro, ${ }^{1}$ Niterói, RJ - Brasil

Pontifícia Universidade Católica do Paraná, ${ }^{2}$ Curitiba, PR - Brasil

Universidade Federal do Maranhão, ${ }^{3}$ São Luís, MA - Brasil

Instituto do Coração (InCor), Hospital das Clínicas, Faculdade de Medicina, Universidade de São Paulo, ${ }^{4}$ São Paulo, SP - Brasil

Universidade Federal de Minas Gerais Faculdade de Medicina, ${ }^{5}$ Belo Horizonte, MG - Brasil

Hospital de Clínicas de Porto Alegre - Serviço de Cardiologia, ${ }^{6}$ Porto Alegre, RS - Brasil

O Departamento de Insuficiência Cardíaca (DEIC), da Sociedade Brasileira de Cardiologia, completou 20 anos de existência em 2020 e representa um robusto legado nas áreas de atividades científicas e associativismo da cardiologia brasileira.

Sua construção representou um marco importante no enfrentamento da insuficiência cardíaca (IC), uma complexa síndrome clínica progressiva e frequentemente fatal. A IC deve ser abordada de forma multidisciplinar, apoiada na ciência translacional e nas boas práticas (diretrizes e protocolos clínicos), envolvendo pacientes, famílias, cuidadores, gestores e toda a sociedade, tendo em vista seus impactos sociais no Brasil e no mundo.

Com o envelhecimento populacional e o aumento da sobrevida dos pacientes com doenças cardiovasculares, a prevalência de IC está aumentando globalmente, com uma estimativa de 26 milhões de pessoas acometidas no mundo, além de milhares de casos não diagnosticados. ${ }^{1}$ A IC é causa líder de hospitalização no mundo, e isso resulta em uma sobrecarga em todos os níveis de cuidado. Estima-se que a IC afeta aproximadamente 2,5 milhões de pessoas no Brasil, e um estudo recente revelou o seu impacto financeiro no país, com um gasto estimado de R \$22,1 milhões/6,8 milhões de dólares no ano de 2015. ${ }^{2}$ Além disso, o estudo mostrou uma substancial perda de bem-estar; dos 521.941 de anos de vida perdidos ajustados por incapacidade, ajustados para comorbidades, há 270.806 de anos de vida saudável perdidos em virtude de incapacidade e 251.941 de anos de vida perdidos em decorrência de morte prematura.

O DEIC foi idealizado a partir da liderança da professora Maria da Consolação Vieira Moreira e teve o apoio do professor

\section{Palavras-chave}

Insuficiência Cardíaca/tendências; Envelhecimento da População; Hospitalização; Pesquisa Médica Translacional; Diretrizes; Produção Científica.

Correspondência: Evandro Tinoco Mesquita •

Universidade Federal Fluminense Hospital Universitário Antônio Pedro - R.

Marquês de Paraná, 303. CEP 24033-900, Niterói, RJ - Brasil

E-mail: etmesquita@gmail.com

Artigo recebido em 10/11/2020, revisado em 19/11/2020, aceito em $02 / 12 / 2020$

DOI: https://doi.org/10.36660/abc.20201200
Gilson Soares Feitosa, na ocasião, presidente da Sociedade Brasileira de Cardiologia (1999-2001), mobilizando líderes de todo o Brasil, com destaque para o professor Edimar Alcides Bocchi, levando à formação do Grupo de Estudos de Insuficiência Cardíaca (GEIC) no ano de 2000. Em 6 de julho de 2001, sob a presidência da professora Maria da Consolação, foi realizado, em Belo Horizonte-MG, o I Simpósio Brasileiro de Insuficiência Cardíaca, por ocasião do XII Congresso da Sociedade Mineira de Cardiologia (Quadro 1).

Com uma trajetória de sucesso, construída por líderes da IC do país, devido ao crescimento do número de associados e relevância na produtividade científica, o GEIC foi se transformando paulatinamente no DEIC, finalmente criado em 2011, na gestão do professor Fernando Bacal. Esse fato importante ocorreu durante o X Congresso Brasileiro de Insuficiência Cardíaca - ao comemorar 11 anos da fundação do GEIC, na cidade de Belo Horizonte-MG (Quadro 1).

Desde sua criação, são realizados, anualmente, congressos de elevada qualidade científica, com intercâmbio internacional e que proporcionam à comunidade médica brasileira aprimoramento do estado da arte do cuidado multidisciplinar e tratamento da IC. Nos últimos congressos, temos tido mais de 1.000 inscritos e cerca de 200 temas livres têm sido apresentados, possibilitando trocas de experiências com especialistas de várias localidades e nomes de referência, do Brasil e do mundo. Em 2020, devido aos impactos da pandemia do novo coronavírus, o DEIC, de forma revolucionária, realizou um congresso virtual - Heart Failure Summit Brazil 2020 -, com a proposta de apresentar e debater os principais avanços que, nos últimos 12 meses, transformaram a IC e serão motivos para mudanças na nossa Diretriz de IC prevista para ser lançada no primeiro semestre de 2021 (Quadro 2).

Cumprindo seu papel científico, o DEIC tem seu importante projeto de Diretrizes e Atualizações, com o objetivo de demonstrar estratégias e propor recomendações baseadas em evidências. A primeira Diretriz de IC, na forma de um consenso, foi publicada em 1992, em uma fase antes da formação do DEIC, realizada em São Paulo sob a coordenação do estimado mestre Dr. Michel Batlouni (Quadro 3).

Destaca-se, aqui, a publicação em 2014, do I Registro Brasileiro de Insuficiência Cardíaca - Aspectos Clínicos, Qualidade Assistencial e Desfechos Hospitalares - BREATHE, organizado pelo Professor Denilson Campos de Albuquerque, projeto que traçou um panorama da IC em pacientes hospitalizados nas diversas 
regiões do país, identificando a aderência da incorporação de métodos diagnósticos e intervenções terapêuticas. Atualmente, está em curso o Registro Brasileiro da Síndrome de Takotsubo, liderado pelo professor Marcelo Westerlund Montera.

O DEIC tem, de forma contemporânea, ampliado sua abrangência técnico-científica, atuando na IC crônica, na IC aguda (sala de emergência/unidade cardiointensiva), na IC avançada (transplante cardíaco/suporte circulatório mecânico), na IC na criança e adolescente e também nas miocardiopatias. Na área das miocardiopatias tivemos o pioneirismo dos professores Marco Aurélio Dias, Francisco Manes Albanesi, Raul Carlos Pareto Junior, Antonio Carlos Pereira Barreto e Charles Mady, que contribuíram para a formação de líderes na IC.

Nos últimos 5 anos, foram criados em importantes áreas temáticas os grupos de estudo: Grupo de Estudos de Transplante Cardíaco e Assistência Circulatória Mecânica (GETAC), Grupo de Estudos de IC na Criança e em Adultos Portadores de Cardiopatia Congênita (GEICPED) e Grupo de Estudos em Miocardiopatias (GEMIC). Dessa forma, estamos nos organizando dentro da moderna visão de construir um ecossistema para colaboração e cooperação inter e multidisciplinar em diferentes subáreas, o que tem sido uma tendência nas sociedades internacionais de IC: HFA-ESC - https://www.escardio.org/Sub-specialty-communities/ Heart-Failure-Association-of-the-ESC-(HFA) - e HFSA (https:// hfsa.org/). (Quadro 4)

Em 2004, foi idealizado o GEIC Jovem, buscando incentivar o desenvolvimento científico e associativo dos jovens cardiologistas interessados na IC. A primeira reunião realizada no Congresso Brasileiro de Insuficiência Cardíaca em Salvador (2004), desde então, vem se transformando a cada ano e agregando novas lideranças, e agora trazendo como foco a inovação e o empreendedorismo aos nossos congressistas.

Reafirmando seu papel social, com ações comunitárias e em políticas de saúde, durante o 73을 Congresso Brasileiro de Cardiologia (2018), o presidente do DEIC, Salvador Rassi, e o diretor científico, Evandro Tinoco Mesquita, oficializaram o Dia Nacional de Alerta contra a Insuficiência Cardíaca, ${ }^{3}$ comemorado no dia 9 de julho. A data foi escolhida por ser o dia do nascimento de Carlos Chagas, o nosso patrono. Esse primeiro "cardiologista moderno" confirma a citação do saudoso professor Nelson
Botelho, pois usou a visão translacional, aproximando a bancada do leito e conectando um olhar sobre a saúde populacional na doença de Chagas. Além disso, criamos a comenda Carlos Chagas do DEIC destinada aos colegas que se destacam nas áreas de ensino/educação, assistência, inovação, atividades e associativismo. Em parceria com a Rede Brasileira de Insuficiência Cardíaca (REBRIC), trabalhamos não somente com o alerta contra a IC, mas também com a aplicação da literacia, construindo ferramentas para o autocuidado e melhora dos desfechos clínicos (https://www.rebric.com.br/).

A próxima década nos traz novos desafios: primeiro, consolidarmos os caminhos para uma nova área de atuação na cardiologia - o especialista em IC. Na presente década, várias iniciativas, alinhadas à visão contemporânea já bem estabelecida em outros países, foram criadas com o objetivo de promover e capacitar especialistas em IC, garantindo a formação com qualidade técnica e científica.

Além disso, um olhar mais amplo sobre prevenção na IC envolve a compreensão do modelo de doença cardiometabólica crônica, ${ }^{4}$ passando a ser fundamental unir hipertensão arterial sistêmica, obesidade, dislipidemia e diabetes melito na gênese, progressão e tratamento da IC. E, por fim, a necessidade do cuidado integral da IC, cooperando medicina da família, geriatras, internistas, intensivistas e paliativistas. Ao lado disso, os avanços da inteligência artificial, ${ }^{5}$ da medicina digital e da genômica vão construindo uma medicina cardiovascular personalizada na IC que transformarão os conceitos de prevenção, diagnóstico e tratamento, conforme tem sido desenvolvido na amiloidose cardíaca e nas miocardiopatias hereditárias. A pandemia por COVID-19 reforça o conceito de cardiovigilância, ${ }^{6,7}$ pois estudos que utilizaram a ressonância cardíaca identificaram que, mesmo em pessoas sem sintomas, há um grau de agressão ao coração e que deverá ser estudado quanto ao risco futuro do desenvolvimento de miocardiopatia dilatada e insuficiência cardíaca sintomática.

Para comemorar os 20 anos de história do DEIC, reunimos as conquistas de nossa brilhante trajetória e destacamos os cardiologistas, cujo trabalho e dedicação foram responsáveis pela excelência e pelo sucesso do departamento. É uma grande honra resgatar e reverenciar o passado, vislumbrando um futuro, com o desafio de buscar novas ideias e de se renovar. (Figura 1).

Quadro 1 - Presidentes GEIC/DEIC

\begin{tabular}{ll}
\hline Primeira Diretoria GEIC & Presidentes GEIC/DEIC \\
\hline Presidente: Edimar Alcides Bocchi & 2000-2001: Edimar Alcides Bocchi \\
\hline Vice-presidente: Denílson Campos Albuquerque & 2002-2003: Edimar Alcides Bocchi \\
\hline Secretário: Fábio Vilas-Boas Pinto & 2004-2005: Fábio Vilas-Boas Pinto \\
\hline Diretora científica: Maria da Consolação V. Moreira & 2006-2007: Nadine Oliveira Clausell \\
\hline Membros da Comissão Científica: & $\mathbf{2 0 0 8 - 2 0 0 9 : ~ M a r c e l o ~ W e s t e r l u n d ~ M o n t e r a ~}$ \\
\hline Evandro Tinoco Mesquita & $\mathbf{2 0 1 0 - 2 0 1 1 : ~ F e r n a n d o ~ B a c a l ~}$ \\
\hline Dirceu Rodrigues de Almeida & $\mathbf{2 0 1 2 - 2 0 1 3 : ~ J o a ̃ o ~ D a v i d ~ d e ~ S o u z a ~ N e t o ~}$ \\
\hline Fernando Bacal & $\mathbf{2 0 1 4 - 2 0 1 5 : ~ D i r c e u ~ R o d r i g u e s ~ d e ~ A l m e i d a ~}$ \\
\hline Marco Aurélio Silva (in memoriam) & $\mathbf{2 0 1 6 - 2 0 1 7 : ~ L u i s ~ E d u a r d o ~ P a i m ~ R o h d e ~}$ \\
\hline Nadine Oliveira Clausell & $\mathbf{2 0 1 8 - 2 0 1 9 : ~ S a l v a d o r ~ R a s s i ~}$ \\
\hline Salvador Rassi & $\mathbf{2 0 2 0 - 2 0 2 1 : ~ E v a n d r o ~ T i n o c o ~ M e s q u i t a ~}$ \\
\hline
\end{tabular}




\begin{tabular}{ll}
\hline Quadro 2 - Simpósio e Congressos GEIC/DEIC & \\
\hline $1^{0}$ Simpósio Brasileiro de Insuficiência Cardíaca & X Congresso Brasileiro de Insuficiência Cardíaca \\
6 de julho de 2001 - Belo Horizonte-MG & 9 a 11 de junho de 2011 - Belo Horizonte-MG \\
\hline I Congresso Brasileiro de Insuficiência Cardíaca & XI Congresso Brasileiro de Insuficiência Cardíaca \\
28 a 30 de novembro de 2002 - Rio de Janeiro-RJ & 31 de maio a 02 de junho de 2012 - Gramado-RS \\
\hline II Congresso Brasileiro de Insuficiência Cardíaca & XII Congresso Brasileiro de Insuficiência Cardíaca \\
21 de novembro de 2003 - São Paulo-SP & 6 a 8 de junho de 2013 - Porto de Galinhas-PE \\
\hline III Congresso Brasileiro de Insuficiência Cardíaca & XIII Congresso Brasileiro de Insuficiência Cardíaca \\
25 a 27 de novembro de 2004 - Salvador-BA & 7 a 9 de agosto de 2014 - Ribeirão Preto-SP \\
\hline IV Congresso Brasileiro de Insuficiência Cardíaca & XIV Congresso Brasileiro de Insuficiência Cardíaca \\
23 a 25 de junho de 2005 - Gramado-RS & 18 a 20 de junho de 2015 - Rio de Janeiro-RJ \\
\hline V Congresso Brasileiro de Insuficiência Cardíaca & XV Congresso Brasileiro de Insuficiência Cardíaca \\
06 a 08 de julho de 2006 - Goiânia-GO & 11 a 13 de agosto de 2016 - Campos do Jordão-SP \\
\hline VI Congresso Brasileiro de Insuficiência Cardíaca & XVI Congresso Brasileiro de Insuficiência Cardíaca \\
28 a 30 de junho de 2007 - Fortaleza-CE & 11 a 13 de maio de 2017 - Gramado-RS \\
\hline VII Congresso Brasileiro de Insuficiência Cardíaca & XVII Congresso Brasileiro de Insuficiência Cardíaca \\
26 a 28 de junho de 2008 - Búzios-RJ & 28 a 30 de junho de 2018 - Goiânia-GO \\
\hline VIII Congresso Brasileiro de Insuficiência Cardíaca & XVIII Congresso Brasileiro de Insuficiência Cardíaca \\
11 a 13 de junho de 2009 - São Paulo-SP & 8 a 10 de agosto de 2019 - Fortaleza-CE \\
\hline IX Congresso Brasileiro de Insuficiência Cardíaca & Heart Failure Summit Brazil 2020 (DIGITAL) \\
10 a 12 de junho de 2010 - Curitiba-PR & 19 de setembro de 2020 \\
\hline
\end{tabular}

\section{Quadro 3 - Consenso e Diretrizes}

- Consenso brasileiro para o tratamento da insuficiência cardíaca - 1992

- Diretrizes e atualizações de insuficiência cardíaca: 1999, 2002, 2005, 2009, 2012, 2018

- Diretrizes de transplante cardíaco: 1999, 2010, 2018

- Diretriz de cardio-oncologia: 2011

- Diretriz de miocardites e pericardite: 2013

- Diretriz de insuficiência cardíaca e transplante cardíaco, no feto, na criança e em adultos com cardiopatia congênita: 2014

- Diretriz de assistência circulatória mecânica: 2016

Quadro 4 - Programas de Residência Médica e Cursos de Especialização em IC Avançada e Transplante Cardíaco

\begin{tabular}{l}
\hline 1. Instituto Dante Pazzanese de Cardiologia \\
\hline Curso de Aprimoramento em Transplante de Coração em Adultos \\
\hline 2. Sociedade Beneficente Israelita Brasileira Hospital Albert Einstein \\
\hline Curso de Aprimoramento em Transplante e Insuficiência Cardíaca \\
\hline 3. Instituto do Coração (Incor) - HC-FMUSP \\
\hline - Programa de complementação especializada - PCE: Insuficiência Cardíaca Congestiva e Dispositivos de Assistência Ventricular \\
\hline - Residência Médica em Transplante de Coração \\
\hline 4. Universidade Federal de São Paulo - UNIFESP \\
\hline Residência Médica da Escola Paulista de Medicina - Ano opcional: Transplante Cardíaco \\
\hline 5. Hospital de Clínicas de Porto Alegre \\
\hline Residência Médica - Ano adicional: Transplante de Coração \\
\hline 6. Instituto de Cardiologia do Rio Grande do Sul/Fundação Universitária de Cardiologia \\
\hline Residência Médica - Ano adicional para Capacitação em Transplante Cardíaco \\
\hline 7. Instituto de Medicina Integral Professor Fernando Figueira - IMIP \\
\hline Programa de Complementação Especializada - COMESP na área de Transplante de \\
\hline Coração e Insuficiência Cardíaca Avançada \\
\hline
\end{tabular}




\section{Carta Científica}

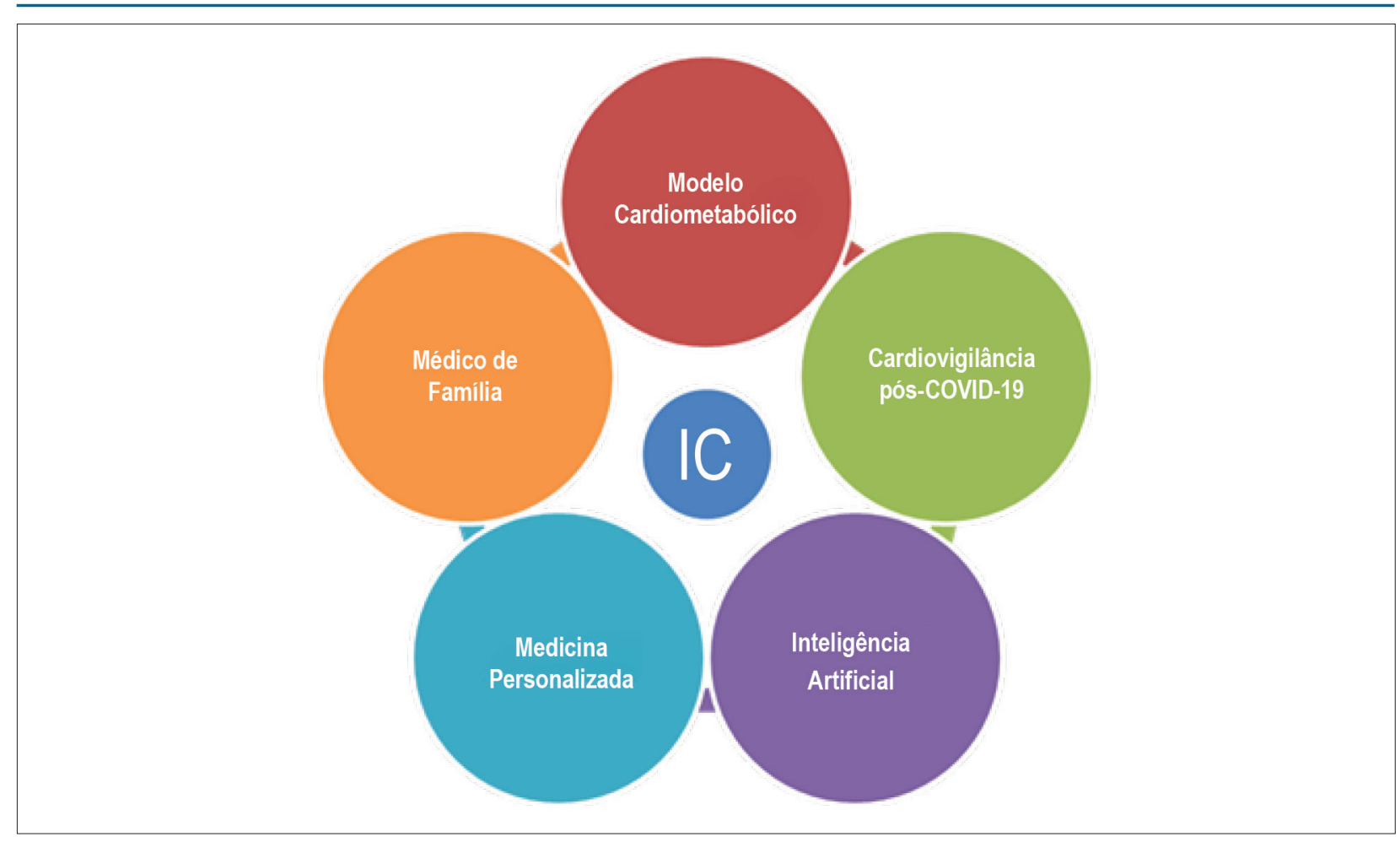

Figura 1 - Desafios do DEIC 2020-30

\section{Contribuição dos autores}

Concepção e desenho da pesquisa e Revisão crítica do manuscrito quanto ao conteúdo intelectual importante: Mesquita ET, Mendes AP, Moura L, Figueiredo Neto JA, Marcondes-Braga FG, Bacal F, Moreira MCV, Clausell NO; Obtenção de dados: Mesquita ET, Mendes AP, Moreira MCV; Análise e interpretação dos dados: Mesquita ET, Mendes AP; Redação do manuscrito: Mesquita ET, Mendes AP, Moura L, Figueiredo Neto JA, Marcondes-Braga FG, Bacal F, Clausell NO.

\section{Referências}

1. Ferreira JP, Krausy S, Mitchellz S, Perel P, Pineiro D, Chioncel O, et al. et al. World Heart Federation Roadmap for Heart Failure. Global Heart. 2019; 14(3):197-214.

2. Stevens B, Pezzullo L, Verdian L, Tomlinson J, George A, Bacal F. Os custos das doenças cardíacas no Brasil. Arq. Bras. Cardiol. 2018; 111(1):29-36.

3. Mesquita ET, Grippa de Souza ALAA, Rassi S. Dia de alerta da insuficiência cardíaca: um tributo ao gênio Carlos Chagas. Arq Bras Cardiol. 2019; 113(1):5-8.

4. Mechanick JI, Farkouh ME, Newman JD, Garvey T. Cardiometabolic-based chronic disease adiposity and dysglycemia drivers. J Am Coll Cardiol. 2019; 75(5):525-38.

\section{Potencial conflito de interesses}

Declaro não haver conflito de interesses pertinentes.

\section{Fontes de financiamento}

O presente estudo não teve fontes de financiamento externas.

\section{Vinculação acadêmica}

Não há vinculação deste estudo a programas de pós-graduação.
5. Souza Filho EM, Fernandes FA, Soares CLA, Seixas FL, Santos AA, Gismondi RA, Inteligência artificial em cardiologia: conceitos, ferramentas e desafios - "quem corre é o cavalo, você precisa ser o jóquei". Arq Bras Cardiol. 2020; 114(4):718-25.

6. Figueiredo Neto JA, Marcondes-Braga FG, Moura LZ, Figueiredo AM, Figueiredo V, Rocha RM, et al. Doença de coronavírus-19 e o miocárdio. Arq Bras Cardiol. 2020; 114(6):1051-7.

7. Fernandes F, Ramires FJA, Fernandes FD, Simões MV, Mesquita ET. Afecções pericárdicas em pacientes com COVID-19: uma possível causa de deterioração hemodinâmica. Arq Bras Cardiol. 2020; 115(3):569-573. 